\title{
Aplicación del método psicoprofiláctico en la Clínica de Maternidad David Restrepo
}

\section{(COMUNICACION PRELIMINAR)}

\author{
Doctores Alberto Cárdenas Escovar, Hugo Campos Garrido, Carlos
}

Alvyar Machado, Bogotá, D. E.

La Clínica de Maternidad "David Restrepo" de Bogotá comenzó a aplicar el método psicoprofiláctico para el parto indoloro en enero de 1959, cuando se preparó un grupo de sesenta pacientes. En marzo, por invitación de la clínica. vino a Bogotá el doctor Pierre Vellay, quien dictó una serie de conferencias sobre el método. El interés suscitado por esta visita se comprende por ser el doctor Vellay una de las personalidades más destacadas en la obstetricia francesa, discípulo del doctor Fernand Lamaze y continuador de su obra, que es adelantar y diİundir el parto sin dolor fundado en un conocimiento científico ce la fisiología de la corteza cerebral, sobre las bases establecidas por la escuela de I. P. Pavlov.

Posteriormente la Clínica Restrepo ha continuado aplicando el método psicoprofiláctico, entendiendo por tal el que acaba de ser mencionado. Se ha adoptado con unidad de doctrina y de práctica y con rigor en la apreciación de los resultados. Se ha. elegido con exclusión de otros, por considerar que el método en cuestión, a diferencia de aquellos, ha dejado la etapa del empirismo y ofrece grandes posibilidades de evolución. Por otra parte, la Clínica Restrepo, en virtud de las características de su organización, es una institución excepcionalmente propicia para la adopción de un método homogéneo de trabajo, que permita 
ei ulterior juicio, con criterios rigurosos y uniformes de los resultados obtenidos. Tal juicio, por fuerza, ha de ser aplazado hasta acumular un volumen respetable de observaciones, y cualquier informe estadístico que la Clínica presente por el momento debe ser tomado con las naturales reservas, como una simple noticia preliminar que sirva de base para discusión e intercambio de ideas.

Actualmente reciben preparación en la Clínica pacientes inscritas en ella para la atención prenatal. Se comienza el entrenamiento alrededor de las 32 semanas de gestación y no se emplea discriminación alguna, de modo que solamente quedan excluídas aquellas que declaran no estar interesadas en el método. Cada curso se extiende por 4 semanas e incluye un promedio de 130 pacientes.

Presentamos a continuación las condiciones en que se realiza el método en la Clínica Restrepo y la manera de calificar los casos.

\section{I.-Descripción del curso}

El acondicionamiento de las pacientes para su trabajo de parto se realiza mediante clases dictadas por los médicos de la Oínica y por preparadoras especializadas.

A cargo de los médicos están los siguientes temas, que se desarrollan ante grupos de 25 a 30 pacientes, acompañadas por sus esposos y en el Salón de Conferencias de la Clínica:

Primera clase.-Presentación del método psicoprofiláctico.

Finalidad del curso que se inicia: educar a la mujer para que se adapte al embarazo y al parto sin dolor.

Presentación de la educación tradicional que había tenido ia mujer para la maternidad.

Educación actual mediante este curso, que consta de tres clases más dictadas por un médico (esquema de ellos) y cuatro clases explicadas por la preparadora.

El esfuerzo personal y la colaboración de la paciente como factores de éxito.

Historia del método: origen, propagación, aprobación por la İglesia Católica. Difusión en Colombia.

Resultados obtenidos y posibilidades para todas las madres.

Segunda clase.-Objeto: conocer los órganos que intervienen en las funciones generativas de la mujer y los procesos que 
se llevan a cabo durante el embarazo. Adaptación del organismo materno durante la gestación. El embarazo como estado normal en la vida femenina.

Mirada de conjunto a las cavidades más conocidas del organismo. Descripción de la cavidad pélvica: periné, orificios. Organos genitales internos.

Descripción y funcionamiento de: trompas, ovarios, útero. Fecundación del óvulo e implantación del huevo.

Desarrollo y evolución intrauterina del niño: placenta, cordón umbilical, membranas ovulares, líquido amniótico.

Higiene del último trimestre del embarazo: oxigenación e importancia de los movimientos respiratorios; dieta; viajes; deportes. Necesidad de un ambiente familiar favorable $y$ actitud que se debe observar frente a un medio mal educado por la tradición.

Tercera clase.-Objeto: estudiar los periodos del trabajo de jarto y el comportamiento que debe tener la paciente durante los mismos.

Estado del cuello uterino y situación del niño en un embarazo a término.

Contracciones uterinas durante el embarazo: medios de corocerlas. Signos de la iniciación del trabajo de parto. Papel del esposo.

Progreso de la dilatación del cuello, gracias a las contracciones uterinas.

Descripción del período de transición: deseo de pujar.

Dilatación completa del cuello uterino.

Comportamiento de la paciente durante el período de dila. tación:

Momento en que debe hospitalizarse.

Primeras contracciones: relajación muscular y respiración lenta y profunda.

Durante la dilatación: relajación muscular y respiración superficial y acelerada.

Período de transición: relajación muscular y respiración superficial, acelerada y soplada.

Nota.-El médico hace una demostración de las variedades de respiración. Antes de continuar con la explicación del período de expulsión se da un pequeño intermedio que sirve para hacer 
notar el cambio radical en el comportamiento de la señora en dicho período.

Periodo de expulsión: descripción de las fases ósea y perineal. Fuerzas necesarias para un correcto desarrollo de la expulsión. Tendencia actual de acortar el periodo de expulsión, colaboración de la paciente.

Conducta de la paciente durante el período de expulsión: €xplicación del mecanismo de la acción de pujar.

Necesidad de obedecer las órdenes del médico: inspire, espire, contenga, agárrese de los barrotes, etc.

Respiración anhelante durante el desprendimiento.

Alumbramiento: contracciones que desprenden la placenta.

Esfuerzo expulsivo para colaborar en el alumbramiento.

Duración del trabajo de parto según la paridad. Valor relalivo del tiempo durante el trabajo; todo depende de la frecuencia e intensidad de las contracciones.

Cuarta clase.-Objeto: apreciar la importancia del cerebro en los procesos de adaptación del sér humano.

Función del sistema nervioso en las relaciones del hombre con el medio ambiente y consigo mismo (Exterocepciones e interocepciones). Los reflejos como respuestas del organismo determinan la conducta del individuo. Reflejos innatos.

Creación de reflejos condicionados por medio de la educación.

Procesos de excitación e inhibición. Objeto de una conducta especial, durante el parto.

Durante el trabajo de parto se van a percibir sensaciones muy propias del mismo.

Instrucciones finales y recuento del comportamiento que debe tener la paciente durante las contracciones del trabajo.

El parto sin dolor no es un parto inconsciente, sino el resultado de un esfuerzo que requiere decisión.

Manifestación del deseo de la Clínica de que todas tengan un parto sin dolor.

Después de cada clase, el médico da a los asistentes la oportunidad de hacer preguntas.

Actualmente la Clínica dispone de 4 preparadoras especializadas, que recibieron su entrenamiento en esta institución y que desempeñan sus funciones según un sistema de turnos, tanto para el adiestramiento de las pacientes como para la vigilancia en salas de trabajo y partos. Las clases a cargo de las preparadoras ocupan cuatro semanas y se inician después de la clase 
cie presentación del método. Asisten por grupos de 12 a 15 pacientes e individualmente se les enseñan los ejercicios que van a utilizar durante el parto.

Primera clase.-La preparadora después de corta explicación, hace una demostración del tema de la clase. Se reparte una hoja con las instrucciones e inmediatamente después cada señora ejecuta los siguientes ejercicios:

Transcribimos a continuación el contenido de dicha hoja:

Entrenamiento de relajación muscular:

10 Acostada de espalda sobre la cama levante ligeramente la cabeza, los brazos y las piernas. Su cuerpo debe permanecer rígido mientras cuenta hasta 5. Afloje a un tiempo todos sus músculos, dejando reposar su cuerpo sobre la cama.

Estos movimientos tienen por objeto enseñarle que la contracción de todos sus músculos a un mismo tiempo hace que su cuerpo permanezca rígido y que la relajación de los mismos lo hace aflojar.

2ọ Acostada de espalda sobre la cama, coloque sus corvas sつbre una almohada, de manera que sus piernas queden cómodamente apoyadas. Afloje todo su cuerpo, relajando voluntariamente sus músculos. Su esposo puede comprobar si su relajación es completa al poder movilizar sus brazos o piernas sin encontrar ninguna resistencia. Lo que debe lograr es que usted misma aprecie si se está relajando completa y rápidamente en el momento que así lo desee.

Nota.-Realizando alternativamente los dos ejercicios anteriores, usted comprobará fácilmente, la notoria diferencia entre la contracción y la relajación musculares.

Los ejercicios siguientes le permiten conocer y controlar di. versos grupos musculares y perfeccionar la relajación completa de sus músculos voluntarios.

Segunda clase.-La preparadora hace un repaso de lo enseñado en la clase anterior y explica los ejercicios respiratorios, haciendo una demostración. Se reparte una hoja con instrucciones cuyo contenido transcribimos:

Ejercicios respiratorios:

10 Respiración profunda y lenta; inspire lentamente hasta llenar por completo sus pulmones. Espire lenta y suavemente, 
como si tratara de inclinar la llama de un 3 vela sin apagarla, hasta vaciar todo el aire de sus pulmones. Repita este ejercicio 5 veces. La respiración profunda y lenta permite distinguir la inspiración de la espiración.

Período del parto en que debe practicar la respiración profunda y lenta; al comienzo del trabajo las contracciones uterinas se presentan cada 15 o 10 minutos; durante cada una de ellas usted debe respirar profunda y lentamente, a tiempo que ejecuta la relajación completa.

2 ? Respiración superficial y acelerada: (m) nsiste en hacer inspiraciones y espiraciones ccotas, tratando de tomar y sacar la menor cantidad de aire. Esta respiración solo debe hacer mover la parte superior del tórax; tanto la inspiración como la espiración deben tener la misma amplitud.

Período del parto en que debe practicar la respiración superficial y acelerada: las contracciones uterinas del parto se van haciendo más frecuentes, así que los intervalos entre una y otra son de 3 a 6 minutos, durando cada contracción hasta un miruto; esto le indica que está en el período de dilatación del cuello y que durante cada contracción debe: hacer una respiración profunda y lenta, seguida de la respiración superficial $\mathrm{y}$ acelerada y al terminar la contracción, una nueva respiración profunda y lenta. Al mismo tiempo debe permanecer completamente relajada.

30 Respiración superficial, acelerada y soplada: consiste en intercalar en la respiración superficial y acelerada un soplo, cada 6 a 8 respiraciones cortas.

Período del parto en que debe practicar la respiración superficial, acelerada y soplada: el deseo de pujar durante las contracciones le indicará que la dilatación del cuello del útero se está completando. USTED NO DEBE PUJAR MIENTRAS EL MEDICO NO SE LO HAYA ORDENADO. En este momento usted debe practicar la respiración soplada para controlar la contracción y evitar el pujo.

Tercera clase.-Tiene por objeto enseñar la manera de pujar en forma efectiva. La preparadora demuestra el esfuerzo del pujo y el momento de hacerlo.

En la hoja que se entrega a cada paciente encontramos:

Acostada de espalda sobre la cama, coloque dos almohadas bajo su espalda de manera que quede ligeramente levantada. Los brazos deben quedar a los lados del cuerpo. A continuación: ins- 
pire profundamente, espire lentamente, inspire profundamente y contenga "el resuello"... Al mismo tiempo tome con cada una de sus manos la pierna correspondiente, retirando los codos cel cuerpo sin apoyarlos en la cama y levante la cabeza. En esta posición podrá hacer el pujo en forma eficaz CONTRAYENDO LOS MUSCULOS DEL ABDOMEN, al mismo tiempo que retiene $\epsilon l$ aire en sus pulmones. Pasado el esfuerzo espire lentamente y relájese.

Período del parto en que debe practicar el pujo: cuando el médico lo ordene.

Mientras está pujando puede necesitar aire nuevo: entonces, sin dejar de pujar, haga una espiración, una nueva inspiración profunda, contenga nuevamente "el resuello" y siga pujando. Cada vez que sienta que una contracción va a aparecer, avise ai médico para que él le ordene el pujo y le recuerde los movimientos apropiados.

Cuando el niño esté "coronando" el médico le dará la orden de NO PUJAR MAS; en este mismo instante le ordenará hacer la respiración superficial y acelerada; cumpla las órdenes del médico sin vacilación, pues así evitará que sus tejidos se desgarren, dejando salir el niño suavemente.

Cuarta clase.-Se dedica a repasar los ejercicios y hacer las correcciones del caso. Se resuelven preguntas y se dan indicaciones sobre la hospitalización. Al final se muestran las salas de trabajo y de parto.

Control de la asistencia: cada paciente recibe un carnet con las fechas y horas de las clases, para registrar el cumplimiento.

Preparación esencial: se considera que una paciente ha recibido la preparación esencial cuando ha asistido a las explicaiones sobre el trabajo de parto y sobre el funcionamiento cereural, así como a todas las clases prácticas.

\section{Atención dell parto}

Llegada la paciente a la Clínica y diagnosticado el estado cel trabajo de parto, se instala en la sala de trabajo y queda bajo el control permanente de la preparadora, quien le recuerda la conducta que debe observar y le hace las correcciones si fueren necesarias. La evolución del trabajo queda bajo el control del médico de turno y del personal de internos. En el periodi expulsivo el médico es el único responsable del comportamiento 
de la paciente y por consiguiente el único autorizado para dar: las órdenes del caso.

La paciente es informada periódicamente del desarrollo de su parto para asegurar en forma completa su colaboración.

Hasta la fecha no se ha permitido la presencia del esposo durante el trabajo, debido a que el comportamiento de algunas pacientes no preparcdas puede presentar inconvenientes, y se tendría que hacer una discriminación, con los consiguientes recla. mos de los no favorecidos.

\section{Calificación de los casos}

Cada paciente es observada durante su parto, por lo menos por tres médicos y una preparadora. La clasificación del caso depende del análisis, según criterios establecidos, por parte del personal que siguió el caso y de las declaraciones de la paciente atendida.

Hasta el mes de agosto de 1959 se empleó, exclusivamente, la calificación de la escuela francesa, preconizada por LAMAZE y VELLAY, pero en vista de la dificultad de englobar en una sola palabra el complejo proceso del comportamiento de la paciente en trabajo, la Sociedad Colombiana de Psicoprofilaxia Obstétrica unificó los criterios de calificación en una historia clínica ¿propiada, adoptando el procedimiento de NICOLAIEV y haciendo a continuación una apreciación global según LAMAZEVELLAY. Esta historia se está utilizando en la Clínica "David Festrepo" y en los institutos afiliados a la Sociedad Colombiana de Psicoprofilaxia Obstétrica.

Presentamos el modelo de historia clínica:

\section{Apreciación de los resultados del método psicoprofiláctio}

(Véanse adelante las instrucciones para llenar este esqueleto de historia clínica).

Nombre:

Edad:

Paridad:

Hisitoria:

Evolución del embarazo:

Fecha:

Antecedentes ginecológicos u obstétricos patológicos:

Antecedentes patológicos generales:

Recibió preparación esencial: $\quad$ SI - NO 
Ingresó a la Clínica en un estado de preparación: BUENO PEGULAR - MALO.

Terminó el curso días antes del parto.

Ingresó a la clínica a las horas de iniciado el trabajo.

Modalidad de la iniciación del trabajo:

CONTRACCIONES.

EXPULSION DEL TAPON MUCOSO.

RUPTURA DE MEMBRANAS.

DOLOR.

HEMORRAGIA.

Estado del trabajo al ingresar a la clínica.

Contracciones ...... Dolores ..... cada ..... minutos

Cuello borrado ...\%, en situación ...... consistencia ...... cillatación ......, centímetros ......

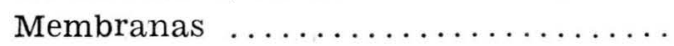

Presentación ......... Altura: $-3,-2,-1,0+1,+2,+3$

Observaciones:

Controla las contracciones con:

RELAJACION MUSCULAR

lenta y profunda

RESPIRACION.... superficial y acelerada soplada

M

Calificación hasta los 3 cmts. de dilatación: horas D. I.

Control de los períodos de dilatación y de transición:

Controla las contracciones con:

RELAJACION MUSCULAR

lenta y profunda

RESPIRACION.... superficial, acelerada soplada

Distocia: $\quad$ SI - NO.

Observaciones

Drogas utilizadas: .en $\ldots$ centímetros de dilatación y a las ..... horas de trabajo ....... en .... centímetros de dilatación y a las .... horas de trabajo.

M

Calificación del período de dilatación: .... horas D.

D. C. $\frac{}{\mathrm{C} \quad \mathrm{Q}}$

Control del período expulsivo:

Distocia: $\quad$ SI - NO. 
Tratamiento:

Drogas utilizadas:

Intervenciones:

Calificación dle período expulsivo: .... horas E. $\frac{\mathrm{M}}{\mathrm{C} \quad \mathrm{Q}}$

Alumbramiento a los ... minutos. Intervención ... SI - NO. Observaciones:

Duración del trabajo:

Analgesia por: DESCONTROL-DOLOR-INCAPACIDAD DE REALIZAR EJERCICIOS-DISTOCIA-INTERVENCION.

Anestesia por: DESCONTROL-DOLOR-INCAPACIDAD DE REALIZAR EJERCICIOS-DISTOCIA-INTERVENCION.

Condiciones del recién nacido: Frecuencia cardíaca .... Tono muscular

Respiración Respuesta estímulo faríngeo Color de la piel

Peso: ........gramos Talla: ....... centímetros

Observaciones: a) del médico; b) de la preparadora, y c) de la paciente.

\section{Calificación total}

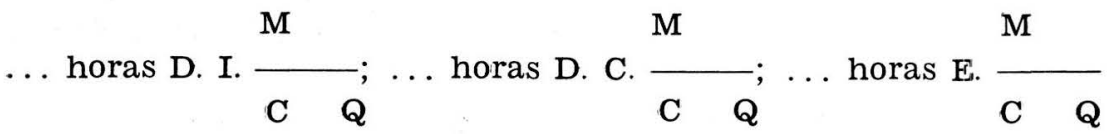

APRECIACION GLOBAL: Excelente; muy bien; bien; regular; pasable; fracaso.

CASO ESPECIAL

Firma del médico

Instrucciones para la valoración compleja de los resultados del método psicoprofiláctico

A/ El período comprendido entre la iniciación del trabajo y los 3 centímetros de dilatación se representa por las letras ...................

D. I.

B/ El período comprendido entre los 3 centímetros de dilatación y la dilatación completa se repre-

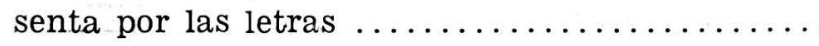

D. C. 
C/ El período de expulsión se representa por la

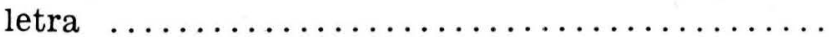

D/ Inmediatamente antes de las letras representativas de cada período se escribe el número que indica la duración en horas del período correspondiente.

E/ En cada etapa se señala la marcha del proceso del parto, así: marcha normal ...............

Marcha complicada ...........

M. N.

M. C.

F/ La conducta objetiva de la paciente observada por el personal, es decir, su inquietud motriz, su posición en la cama, su mímica, etc., se designa con la letra $\ldots \ldots \ldots \ldots \ldots \ldots \ldots \ldots \ldots \ldots$.

G/ Las opiniones de la paciente sobre la indolorización o sus quejas por dolor se designan con la

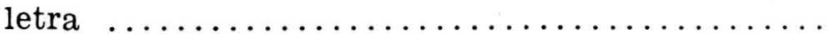

Nota.-Al lado de las letras C y Q se indica el punto de apreciación, según los siguientes criterios:

C $\mathbf{5}=$ paciente activa, comunicativa, realiza fácilmente los métodos aprendidos, no tiene ringuna inquietud motriz $o$ anímica y hasta está propensa a réplicas y bromas.

C $4=$ paciente que no revela inquietud motriz o anímica, pero está concentrada en realizar el létodo; trata de no distraerse, parece atada a sus ejercicios; la aparición de la inquietud se elimina fácilmente al cumplir las insinuaciones del médico o de la preparadora.

c $3=$ paciente que revela inquietud motriz y anímica, sobre todo inquietud cuando el personal se aleja; la inquietud se elimina con las indicaciones del personal o al corregir una distocia que se está presentando. Estas inquietudes no deben ocupar más de 3 horas del total del trabajo de parto.

C 2 = la paciente tiene inquietud motriz y anímica, como en las pacientes no preparadas; pierde el control y no se corrige por la intervención y la ayuda del personal durante gran parte del trabajo. 
C 1 = conducta histeroide de la paciente; sumamente intranquila, gritos, ausencia de contacto con el personal, rechazo de las indicaciones e incluso reacciones negativas a ellas.

Q $5=$ no hay ninguna queja por parte de la paciente.

$Q 4=$ no hay quejas, pero refiere sensaciones desagradables e inquietantes, aunque tolerables, que desaparecen al ejecutar los ejercicios apropiados.

Q $3=$ de vez en cuando aparecen quejas por dolores tolerables o por dolores fuertes que desaparecen al ejecutar los ejercicios por su propia voluntad y sobre todo por la ayuda activa del personal. Los dolores no aparecen en total por más de tres horas del parto.

Q 2 = quejas fuertes, como en las pacientes no preparadas; no se suprimen por la ejecución de los ejercicios enseñados.

$\boldsymbol{Q} 1=$ quejas de tipo histérico durante el trabajo, prudentemente observado, con ignorancia completa y abandono de los ejercicios, quejas que obligan al uso de los analgésicos.

La apreciación global se hará según los criterios preconizados por Lamaze-Vellay.

Excelente: ausencia completa de dolor durante el trabajo; la paciente requiere un esfuerzo similar al necesario para realizar cualquiera de sus ocupaciones diarias.

Muy bien: ausencia completa de dolor durante el trabajo, sin embargo, la paciente requiere un esfuerzo constante para adaptarse a los períodos del parto.

Bien: ausencia de dolor durante el trabajo con ligeros momentos de descontrol.

Regular: paciente con sensaciones dolorosas atenuadas durante el trabajo; ligeros momentos de descontrol.

Pasable: excitación psimotriz durante las contracciones, pero el comportamiento se diferencia del de las no preparadas.

Frcaaso: paciente con sensaciones dolorosas y comportamiento que no se diferencia del de las pacientes no preparadas.

H/ Calificación de las condiciones del recién nacido: se sigue el sistema de Apgar, que considera cinco condiciones, cada una 
REVISTA COLOMBIANA DE OBSTETRICIA $Y$ GINECOLOGIA 379

de las cuales representa dos puntos en caso de estar normal, para un total de 10.

I/ Se llamará Caso Especial aquel que no se acomode en su totalidad a la historia o que según el criterio del médico deba ciestacarse aparte.

Según estas líneas generales se está efectuando en la Clínica "David Restrepo" la educación por el método psicoprofiláctico. La experiencia va mostrando los errores que deben corregirse $y$ las ideas nuevas que permiten mejorar los resultados. Como so dijo al principio de este artículo, ulteriomente se hará una co. municación sobre el material estadístico, una vez que éste al. cance un volumen conveniente

N. de la R.-Al dar cabida en el presente número a la anterior "comunicación preliminar", tratamos de estimular el entusiasmo inicial de uno de los Equipos de Sicoprofilaxia de más reciente fundación en el país. 